\title{
The NFVRG Network Function Virtualization Research at the IRTF
}

\author{
D. Lopez ${ }^{1}$ and R. Krishnan ${ }^{2}$ \\ ${ }^{1}$ Telefónica $I+D$, Spain \\ ${ }^{2}$ Dell, USA \\ E-mail:diego.r.lopez@telefonica.com; ramki_krishnan@dell.com
}

Received 15 April 2015;

Accepted 01 May 2015

\begin{abstract}
This paper presents the recently created NFVRG within the IRTF, outlining the story of its constitution, describing and analysing its charter, and presenting the results achieved so far (in despite of its short lifetime) and the intended goals, especially in the short-mid-term.
\end{abstract}

Keywords: NFV, research, IRTF, IETF, SDN, virtualization.

\section{Introduction}

The concept of a network essentially implemented in software is a general trend that has consolidated in the recent years and that we can consider based on two main principles:

- Providing general software interfaces for the configuration and usage of network resources thus abstracting the complexity and deployment details of actual network infrastructures.

- Decoupling the different planes conforming the network, and using open interfaces between them, in order to make the supporting infrastructure

Journal of ICT, Vol. 3, 57-66.

doi: $10.13052 /$ jicts2245-800X.313

(c) 2015 River Publishers. All rights reserved. 
as much regular and homogeneous as possible, and relying on software mechanisms to support specialized functionalities.

According to these principles, network services are provided by a layered structure, grounded on general-purpose, homogenous hardware, with one or several software layers running on top of it and defining network behavior and functionalities in general. Everything running on the network, from basic functionalities to user applications, and including management and operation elements, becomes a software module that uses the open interfaces provided at each layer.

There are two essential directions that can be applied independently, though they greatly benefit from their simultaneous application, to the degree of being suitable to be considered essentially intertwined. Software Defined Networking (SDN) goes for the decoupling of the control and data planes in a network to gain programmability and simplify data plane elements, while Network Functions Virtualization (NFV) goes beyond, advocating for the general separation of functionality (on software) and capacity (on a general virtualization layer running upon standard regular hardware), increasing network elasticity and drastically reducing the heterogeneity of the supporting infrastructure.

SDN and NFV have been termed as not properly networking technologies, but tools, useful but not at the core of network themselves. While this is arguably true for the application of software to current networking practices, it is also widely acknowledged that they have a strong potential of transforming the way in which networks are designed, deployed and managed. The IETF, focused on the engineering of the Internet through standardization, is exploring the applicability of these technologies as tools to improve different areas of network provisioning and management. However, from the view of the IRTF [1], precisely dedicated to explore the impact of new technologies in the future of Internet, it was natural to address both the long-term implications of these technologies, as well as contribute to consolidate them as essential tools for supporting the general evolution of the network. Given that the concept of SDN predates NFV, the SDNRG [2] was the first created, and the brand new NFVRG followed it.

This paper describes the story of the NFVRG and its consolidation within the IRTF, analyzes in detail its current charter and the connection with other activities related to NFV standardization and development, and details the results achieved so far and the future evolution we envisage for the group. 


\section{The NFVRG Story}

From their initial stages back in 2012, the NFV proponents were well aware of the need of strong research activities and direct collaboration with the research communities to foster a proposal that, while based on the solid ground of current well-established cloud principles, was blazing the trail in a completely new application scenario of these principles. The first NFV whitepaper [3], where the very acronym was coined, lists among the potential benefits of NFV the possibility of a more open and efficient innovation cycle for network infrastructures, where research results could be brought into operational practice in a much shorter time than the current approaches.

The second NFV whitepaper [4], released one year after, directly encouraged "to create new applied research and study programs around NFV". During that year the term NFV had widely spread, and papers, special sessions and workshops started to appear elsewhere. The NFV community formulated a Research Agenda by the beginning of 2014 [5] that included the research topics that were considered most relevant, and that constituted the base for the further NFVRG charter. The first direct references to NFV in the IRTF appeared along the SDNRG meetings at IETF86 in Orlando [6], while the IETF first formally considered NFV during the discussions on the creation of the SFC WG at IETF87 in Berlin [7]. A first introductory session on NFV goals and first steps was held at IETF88 in Vancouver, during a lunch ad-hoc panel discussion. With the increasing of NFV-related research activities, the SDNRG became the natural place for discussing its implications for Internet architecture and protocols at the IRTF, and therefore the number of NFV contributions to the group grew.

The NFV community had started to appoint liaison officers to the most relevant standards organizations in order to foster collaboration, and during IETF89 in London there were several informal discussions among the appointed NFV liaisons to the IETF and the IETF and IRTF leadership. As a result of these discussions, the idea of a potential research group focused on NFV took shape, and an initially small group of people started to sketch the idea, preparing a first proposal for a charter that was shared with the IRTF Chair. After some initial discussion, the IRTF Chair agreed to start NFVRG as a "proposed RG", a tentative status that is used to gauge community interest and potential results of proposed research groups within the IRTF. That allowed the NFVRG proponents to call for a first meeting at IETF90 in Toronto, scheduled out of the agenda along one of the lunch breaks, and 
a subsequent in-agenda one at IETF91 in Honolulu [8], as well as a much stronger interaction with the IRTF community at large. Two interim meetings were also organized with the main goal of refining the original charter.

The two meetings as proposed RG gather a high participation at all levels: audience, presentations and discussions among the participants. The IETF datatracker has listed eight Internet-drafts directly associated with the proposed RG [9] and the charter was gaining in maturity through direct discussion in the NFVRG mailing list. By the end of 2014, the current co-chairs made a direct request to the IRTF Chair to formally charter the NFVRG as an IRTF Research Group. The announcement of the formal charter of NFVRG was made by the end of January 2015.

\section{The NFVRG Charter in Detail}

The charter of the NFVRG as approved by the IRTF is available at [10]. It discusses the general goals and challenges of NFV research, with special emphasis on its potential impacts on the Internet architecture. As said above, it was originally inspired on the NFV Research Agenda released by the ETSI NFV ISG Technical Steering Committee.

The charter starts with a general declaration of what NFV is and the main characteristics and expected benefits of this technology. Three particular aspects are especially relevant, in defining the main directions for the future work of the NFVRG. First, the possibility of re-thinking current network functions out-of-the-box, building virtual topologies associated with strict functional criteria and fully abstracted from the underlying infrastructure topology, especially in what relates to the constituent nodes. Second, the opportunities for new service provision patterns, where the decoupling between functionality and capacity will enable new roles and new business models. Third is re-thinking the way service provider points-ofpresence [13] are managed by exploring opportunities for global optimization across multiple sub-systems beyond networking leading to substantial OPEX savings. While the base technologies (cloud infrastructures and network virtualization essentially) are reasonably established, their application to the provision of network functions is not, and this application domain poses additional challenges that needs to be explored by the research community. The relevance of other research activities within the industry and academia is acknowledged, with a commitment to open collaboration of the NFVRG with technical workshops and scientific conferences, and technology publications. 
Besides the consideration of general research problems and specific contributions to standardization activities within IETF WGs, NFVRG will play an important role in contribution of research findings to the standardization efforts in the ETSI NFV ISG, providing exploratory guidance and experimental evidence. Additionally, the NFVRG will help in driving the NFV architectural vision for open source projects in OpenStack [14], OpenDaylight [15], and OPNFV [16] etc. by complementing the NFV standardization efforts in ETSI. Let us consider here and detail the areas of interest included in the NFVRG charter:

- Exploring the new network architectures that can be based on virtualized network functions (VNFs), with a special emphasis in what we could call "network function deconstruction", exploring how to build these functions from virtualized components and identifying compositional patterns.

- How NFV challenges the current cloud architectures, especially in what relates to data plane workloads and the need to adapt VNF deployment to satisfy service topologies and mobility requirements.

- New patterns in network and service function chaining, the architecture implications for chains and the deployment implications for service paths. Extremely relevant issue here are the design and implementation patterns related to virtual and non-virtual functions chaining, and the possibilities of automating it.

- A key aspect of NFV is the orchestration of the virtualization aspects, so the scalability and flexibility associated with cloud-like infrastructures can be achieved. Autonomous orchestration and optimization of VNFs and network services based on them constitute an essential aspect to keep the orchestration problem within manageable limits.

- Reliability and failure characterization is one of the main concerns expressed by many network practitioners when faced with NFV, and thus the NFVRG will study the requirements and mechanisms to ensure reliable virtualized network functions and services.

- NFV will support new operational models, posing a challenge to current practices in network service provider OSS (operation support systems), as well as opening the possibility to streamline them and fostering the application of DevOps [11] paradigm to network operation.

- Virtualized network functions and the infrastructure they run upon will require appropriate abstractions and domain-specific languages to support those levels of abstraction and the openness of the corresponding 
interfaces. These include the exploration of such abstractions and the supporting languages and APIs, frameworks for combined processing, network and storage description, policy languages...

- It is clear that there will be no NFV zero day, and there is a need to address heterogeneous networks, what in NFV terms implies the coexistence with non-virtualized infrastructure and services, especially when it has to do with management of this heterogeneity and the exploration of the most efficient migration paths.

- Virtualized network functions are expected to play an important role in the convergence of different network technologies and application domains (wired, optical, cellular, satellite...) and they have been acknowledged as an essential component of the so-called 5G networks [12].

- Network security concepts are based to a great extent in physical access control (just think about the perimeter concept in current firewalling practice), and this has been brought to the extreme in the case of network infrastructures, where security procedures rely explicitly and even implicitly in the limited physical access to links and nodes. The advent of NFV will shake these assumptions, especially when the network function deconstruction mentioned above is applied. Research in new mechanisms for security, trust and attestation are therefore essential to bring NFV to its full potential.

- Another big hurdle in the NFV path are the claims about inferior performance of VNFs with respect to their physical, traditional counterparts, what NFV proponents reply with the argument that VNFs should only be required to be good-enough for their particular purposes. This implies the need for appropriate performance modeling and characterization.

- Running network functions on a virtualized infrastructure will allow a much more complete and flexible monitoring and data collection (probes can be seamlessly deployed), as well as rely on those collected data to perform adaptive orchestration and management, so real-time big data analytics and data-centric management will be of direct applicability.

- When it comes to this smart management and orchestration, energy efficiency has a specific relevance by its own, especially considered in terms of end-to-end and system-wide optimization of the whole infrastructure (compute, storage and infrastructural network). 
- Last but not least, NFV brings the promise of new business models and a deep change in the network service provision economics.

\section{Current Results and Future Actions}

As we said when outlining the story of NFVRG, in order to focus the activity to maximize impact and address the most pressing research issues, the group has agreed on a few work items to be considered for the near term and therefore fostered by the chairs of the group. This does not imply that contributions addressing any other area in the charter are discouraged, but as an indication of the priorities of the group in the coming months, where results (in the form of research Internet drafts or contributions to other groups) should be produced. These work items are:

1. Policy-based resource management (as depicted in Figure 1 below), addressing optimized resource management and workload distribution based on policy.

2. Analytics for visibility and orchestration, contemplating techniques for the applicability of real-time analytics not only to providing visibility into the NFV infrastructure but also to optimizing resource usage for the purposes of orchestration.

3. Performance modeling focused on facilitating the transition to NFV, so an equivalence model between physical and virtual network functions can be established.

4. Service verification in what relates to security and resiliency, considering aspects related to service and function attestation, and service protection based on elasticity and dynamic management.

Along the two meetings run under the proposed NFVRG, more than twenty presentations were made with lively discussions and a high audience, above 150 attendants. 8 drafts have been submitted, mostly addressing the nearterm work items, and several of them are progressing along the IRTF track. Direct links have been established not only with other bodies in the "natural" environment of NFVRG (the IETF and the IRTF as well as the ETSI NFV ISG), but also with open-source projects like OpenStack and the recently launched OPNFV.

As a brief summary, we conclude that we have a huge amount of work ahead with our brand new NFVRG, but it promises to be a more than exciting job. And the recruiting office is always open to those willing to join the (select) club of NFVRGers. 
64 D. Lopez and R. Krishnan

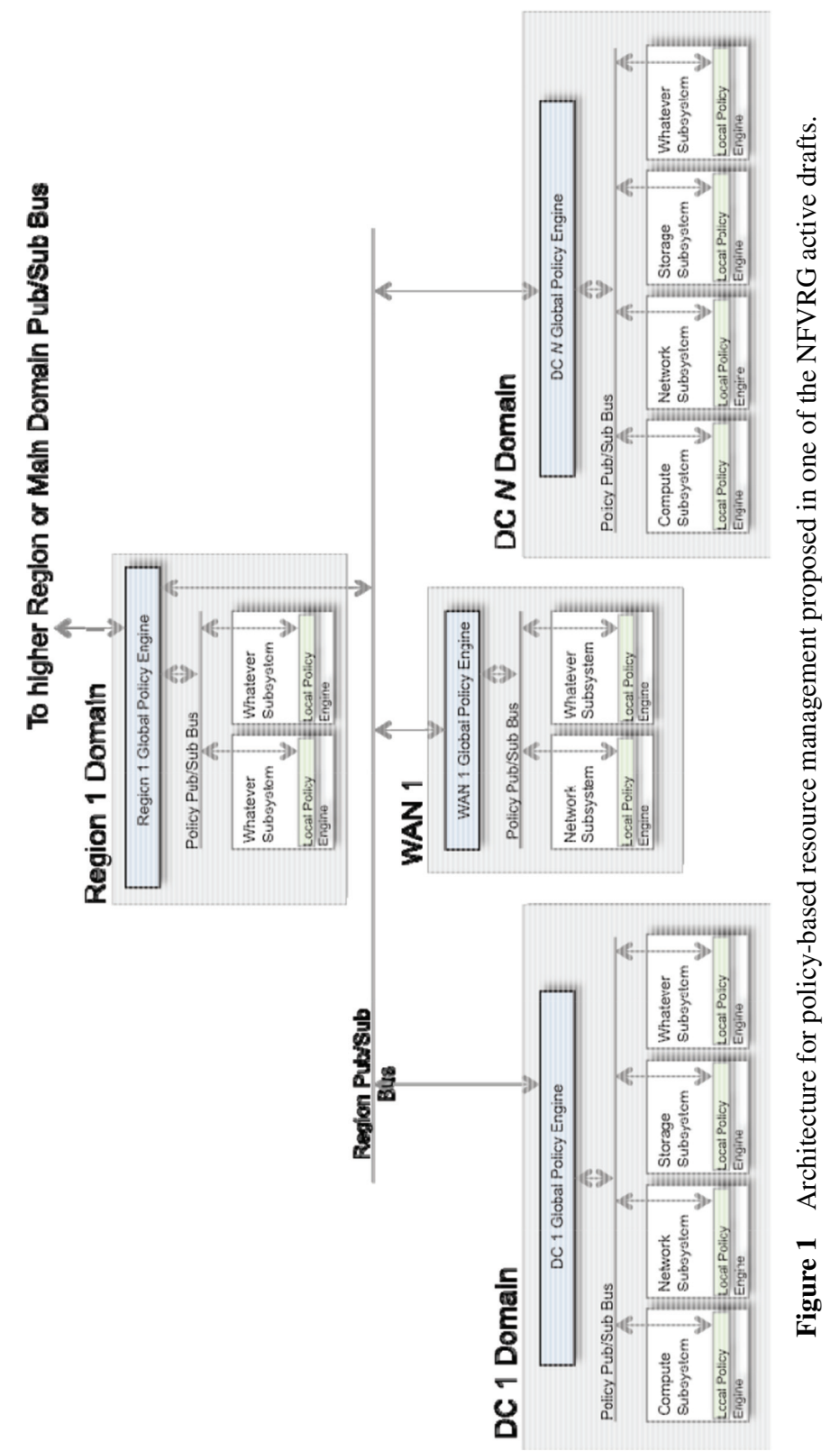




\section{References}

[1] "Internet Research Task Force (IRTF)" https://irtf.org/

[2] "Software-Defined Networking Research Group (SDNRG)" https://irtf .org/sdnrg

[3] "Network Functions Virtualisation - Introductory White Paper" https:// portal.etsi.org/nfv/nfv_white_paper.pdf

[4] "Network Functions Virtualisation - Update White Paper" https://portal .etsi.org/nfv/nfv_white_paper2.pdf

[5] "ETSI NFV ISG proposed topics for Research Agenda 2014" https: //portal.etsi.org/Portals/0/TBpages/NFV/Docs/NFV_Research_Agenda_ 2014.pdf

[6] "Network Functions Virtualisation" http://www.ietf.org/proceedings/86/ slides/slides-86-sdnrg-1.pdf

[7] "Network Functions Virtualization (NFV)" http://www.ietf.org/proceedin gs/87/slides/slides-87-nsc-0.pdf

[8] "NFVRG - IETF 91 Honolulu" http://www.ietf.org/proceedings/91/nfvrg .html

[9] "NFVRG - current IRTF drafts" https://datatracker.ietf.org/rg/nfvrg/docu ments/

[10] "NFVRG charter," https://irtf.org/nfvrg

[11] Debois, Patrick. "Devops: A Software Revolution in the Making?" Cutter IT Journal.

[12] "NGMN 5G White Paper - Executive Edition by NGMN Alliance" http://www.ngmn.org/uploads/media/141222_NGMN-Executive_Versio n_of_the_5G_White_Paper_v1_0_01.pdf

[13] "ETSI NFV Terminology for Main Concepts in NFV" http://docbox.etsi .org/ISG/NFV/Open/Published/gs_NFV003v010101p\%20-\%20Termino logy.pdf

[14] "OpenStack open source project" https://www.openstack.org/

[15] "OPNFV open source project" https://www.opnfv.org/

[16] "OpenDaylight open source project" http://www.opendaylight.org/ 


\section{Biographies}

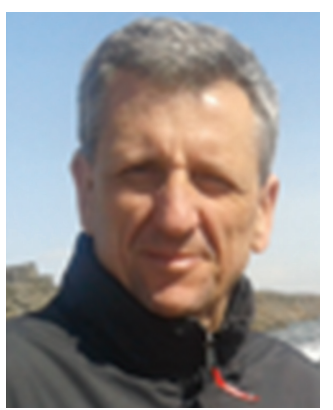

D. R. Lopez is a Senior Technology Expert on network middleware and services within the GCTO Unit of Telefónica I+D. Diego is currently focused on identifying and evaluating new opportunities in technologies applicable to network infrastructures, and the coordination of national and international collaboration activities. Diego is actively participating in the ETSI ISG on Network Function Virtualization (chairing its Technical Steering Committee), the ONF, and the IETF WGs connected to these activities, acting as co-chair of the NFVRG within the IRTF.

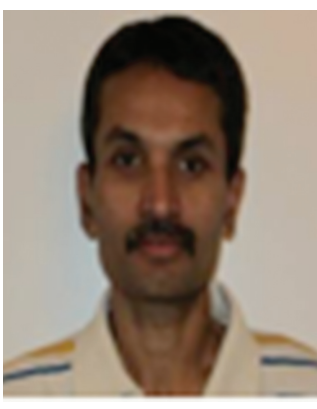

R. Krishnan is an industry expert in the area of Networking and currently Distinguished Engineer and CTO of NFV in Dell. He is a recognized innovator with 20+ US patents and several conference papers. He is co-chair of NFV Research Group in IRTF. He is also a thought leadership speaker in conferences such as ONS, OpenStack, and OpenDaylight etc. 